\title{
Impact of marker density on the accuracy of association mapping Weihua Zhang* ${ }^{* 1,4}$, Winston $\mathrm{Lau}^{\dagger 2}$, Cheng $\mathrm{Hu}^{3}$ and Tai-Yue $\mathrm{KuO}^{2}$
}

Address: ${ }^{1}$ Section of Cancer Genetics, The Institute of Cancer Research, 15 Cotswold Road, Belmont, Sutton, Surrey SM2 5NG, UK, ${ }^{2 H u m a n}$ Genetics Division, Duthie Building (Mailpoint 808), Southampton General Hospital, University of Southampton, School of Medicine, Tremona Road, Southampton, SO16 6YD, UK, 33Shanghai Diabetes Institute, Shanghai Jiaotong University, 600 Yishan Road, Shanghai 200233, People's Republic of China and ${ }^{4}$ Department of Cardiology, Ealing Hospital NHS Trust, Uxbridge Road, Southall, Middlesex, UB1 3HW, UK

Email: Weihua Zhang* - weihua.zhang@eht.nhs.uk; Winston Lau -wwsl@soton.ac.uk; Cheng Hu - alfredhc@sjtu.edu.cn; Tai-

Yue Kuo - kuotaiyu@soton.ac.uk

* Corresponding author †Equal contributors

from Genetic Analysis Workshop 15

St. Pete Beach, Florida, USA. II-15 November 2006

Published: 18 December 2007

BMC Proceedings 2007, I (SuppI I):SI66

This article is available from: http://www.biomedcentral.com/I753-656I/I/SI/SI66

(C) 2007 Zhang et al; licensee BioMed Central Ltd.

This is an open access article distributed under the terms of the Creative Commons Attribution License (http://creativecommons.org/licenses/by/2.0), which permits unrestricted use, distribution, and reproduction in any medium, provided the original work is properly cited.

\begin{abstract}
We studied the impact of marker density on the accuracy of association mapping using Genetic Analysis Workshop 15 simulated dense single-nucleotide polymorphism (SNP) data on chromosome 6. A total of 1500 cases and 2000 unaffected controls genotyped for 17,820 SNPs were analyzed. We applied the approach that combines information from multiple SNPs under the framework of the Malecot model and composite likelihood to non-overlapping regions of the chromosome. We successfully detected the associations with disease Loci $C$ and $D$ and predicted their locations as small as zero distance to Locus C when it was "typed" and II $2 \mathrm{~kb}$ from the untyped rare Locus D. Reducing marker density decreased the accuracy of location estimates. However, the predicted locations were robust to variations in the number of SNPs. Generally, the linkage disequilibrium (LD) map reflecting distances between markers in relation to LD produced higher accuracy than the physical map. We also demonstrated that SNP selection based on equal LD distance outperforms that based on equal physical distance or SNP tagging. Furthermore, ignoring rare SNPs diminished the ability to detect rare causal variants.
\end{abstract}




\section{Background}

As the cost of genotyping decreases, genome-wide association (GWA) mapping of the predisposition genes for complex diseases is becoming a common study design in genetic epidemiology. As the huge number of singlenucleotide polymorphisms (SNPs) in the human genome is still prohibitive for exhaustive investigation, subsets of SNPs have often been selected for large scale studies. Morton et al. developed a novel GWA mapping approach based on the Malecot model and composite likelihood combining multiple marker information from non-overlapping genomic regions to predict the locations of disease variants [1]. We applied this approach to the Genetic Analysis Workshop (GAW) 15 Problem 3 simulated dense chromosome 6 data with the knowledge of the answers and we studied the effect of SNP density on the accuracy of association mapping.

\section{Methods \\ Data}

The simulated data set contained 1500 families with a sib pair affected with rheumatoid arthritis (RA) and a random sample of 2000 unrelated and unaffected individuals. To form a case-control study, we selected the first sibling per family as a case. A total of 1500 cases and 2000 controls from Replicate 1 were analyzed. There are three simulated disease loci. HLA-DR is at the same location of 32484.648 $\mathrm{kb}$ as Locus C, where a SNP denseSNP6_3437 lies, so we considered this SNP the disease variant C. Locus D is at $37233.784 \mathrm{~kb}$, in very weak linkage disequilibrium (LD) with Locus $\mathrm{C}$. The minor allele frequency (MAF) for the $\mathrm{C}$ allele was 0.4055 in control samples. The $\mathrm{D}$ allele has a population frequency of 0.0083 , but the variant was not typed.

Genotype data were composed of 17,820 SNPs on chromosome 6, mimicking a $300 \mathrm{~K}$ GWA scan with no missing values. Fifty-eight SNPs showing departure from HardyWeinberg equilibrium (HWE) in control samples $\left(\chi^{2}{ }_{1} \geq\right.$ 10 for either Pearson's or likelihood ratio chi-square tests) were discarded [2]. Following convention, 2061 rare SNPs with $\mathrm{MAF}<5 \%$ were further removed except when otherwise indicated. The main data set (1) was thus composed of a total number of 15,701 SNPs. In another experiment we retained all SNPs but removed 26 SNPs showing departure from HWE by the likelihood-ratio test and this generated 17,794 SNPs (data set 2).

\section{LD map}

The physical map length was $170,813 \mathrm{~kb}$. LD maps expressed in LD units (LDUs) were constructed based on pair-wise LD for multiple markers in control samples [3]. LDU is the product of $\varepsilon$ and $\mathrm{kb}$ distance for an interval of two adjacent SNPs and is additive, where $\varepsilon$ represents the exponential decline of LD with distance for that interval.
We used the LDMAP-cluster, a parallel version of LDMAP program that rapidly constructs the maps of equally divided chromosome segments http:www.som.soton.ac.uk/research/geneticsdiv/ epidemiol ogy/ldmap/[3]. For each segment, an overall $\varepsilon$ value was also estimated. The LD map length was 1311.225 LDUs for the main data set and 1237.923 LDUs for data set 2. SNPs can have the same LDU if they are in an LD block. Therefore, we also made tilted LD maps by reassigning LDU locations for the SNPs with the same LDU by linear interpolation.

\section{Association mapping}

A chromosome is divided into non-overlapping consecutive regions of a minimum number of 30 SNPs and a minimum length of 10 LDUs by default without breaking LD blocks. Each genomic region was then analyzed separately. Association between SNP alleles and disease status in the Malecot model is a function of several parameters. Composite likelihood combines information of all marker-disease association in a genomic region. The parameters were estimated through fitting the model to the data with a map in LDU or kilobases and by minimizing -2 natural log composite likelihood (denoted as $\Lambda$ ) [1]. The estimated location $S$ of the disease locus is converted to a kilobase scale. The significance test is performed by contrasting two hierarchical models. Model A assumes no association with the disease, therefore $S$ is not estimated. Model D assumes an association with the disease and $S$ and two other parameters are estimated and $\varepsilon$ is specified. The difference in $\Lambda$ between models $A$ and $D$ $\left(\Lambda_{\mathrm{A}}-\Lambda_{\mathrm{D}}\right)$ is monotonic to the magnitude of chi-square with three degrees of freedom $\left(\chi^{2}{ }_{3}\right)$. Permutation by shuffling case-control status for each region was performed to obtain empirical $p$-values [1]. The algorithms were implemented in the CHROMSCAN program. A parallel version, CHROMSCAN-cluster, deployed on a local Beowulf cluster http://www.som.soton.ac.uk/research/geneticsdiv/epi demiology/chromscan/ was used for computing 1000 replicates.

The values of $\varepsilon$ were obtained by averaging over eight segments in LD map construction, which were 1.14472 and 0.00568 for LD and kilobase maps, respectively, for the main data set, and 1.14386 and 0.00544 over nine segments for data set 2 . Theoretically, a more accurate $\varepsilon$ may be obtained by fitting the maps to the whole chromosome data, but the extensive computing power required for the task is impractical to implement and beyond the current computing resource. Also, slightly altered $\varepsilon$ values did not appear to have an appreciable effect (data not shown).

For comparison, a single SNP $\chi^{2}{ }_{1}$ was obtained by the $2 \times$ 2 allelic count table and the most significant SNP (msSNP) showing maximal $\chi^{2}{ }_{1}$ in each region was identi- 
fied. Location error (in kilobases) was defined as the difference between $S$ or the location of the msSNP and the true location of disease variant. Accuracy refers to the precision of the predicted location $S$. The smaller the error, the higher the accuracy.

\section{SNP density}

To generate different SNP density, we selected every $i^{\text {th }}$ SNP $(i=2,3, \ldots, 20,25,30)$ in the order of their physical locations from the full data set, representing $1 / i$ the number of SNPs in the original set. For a candidate region spanning Loci C and D with rare SNPs included, we used Tagger implemented in the Haploview software to select tagging SNPs that optimally capture allelic variation among SNPs at a given $r^{2}$ threshold based on pairwise LD in control samples [4]. For comparison, we selected the same number of SNPs as Tagger but in equal LDU or kilobase distance. To do this we used the tilted LD map in which every SNP had a unique LDU location. We also studied the impact of region length and sample size.

\section{Results and discussion}

\section{Association mapping of disease loci in full data set}

Fourteen out of 126 regions showed nominal significant association with RA $(p<0.05)$, among which eight consecutive regions spanned Loci $\mathrm{C}$ and $\mathrm{D}$ (Table 1). Five regions remained significant after Bonferroni correction, among which four surrounded or spanned Locus $\mathrm{C}$, and one covered Locus $\mathrm{D}$ (Table 1). Locus $\mathrm{C}$ was inside the most significant region 29. Therefore, the three regions surrounding Locus $\mathrm{C}$ with less significance levels must be the result of $\mathrm{LD}$ between variant $\mathrm{C}$ and other SNPs. The discontinuity of significance surrounding region 32 indicated that this region harbored another disease locus and indeed, this was where Locus D lies. Therefore, we successfully detected Loci $\mathrm{C}$ and $\mathrm{D}$ in the initial analysis. The lowest $p$ for the rest of the regions was 0.0064. Given that there were no other disease loci, the approach had a right type I error rate $(6 / 118=0.05)$. A lesson learned was that when there was long-range $\mathrm{LD}$, consecutive regions show- ing association may reflect one instead of several disease loci. As an alternative to merging regions, we studied the impact of region length on accuracy (see below).

$S$ for Locus C was reasonably accurate (55 kb apart from true location using LD map). However, the location error was $542 \mathrm{~kb}$ for Locus D and the 95\% confidence interval did not include Locus D. Removing 10 SNPs showing significant $\mathrm{LD}$ with variant $\mathrm{C}$ did not change the results. We then divided region 32 into two or three sub-regions. Again, we did not detect significant association in the middle part where Locus D lies, although we detected the associations in the first and third sub-regions where two clusters of highly significant SNPs lay. Because Locus D is rare, the removal of rare SNPs may have had an effect. We then added rare SNPs and used the corresponding LD map and $\varepsilon$ values, and the location accuracy was markedly improved for Locus D (Table 2). Among the added rare SNPs, three were highly associated with the disease: denseSNP6_3931,_3933, and SNP6_162 $\left(\chi^{2}{ }_{1}=118,116\right.$, and 116 , respectively). It is therefore a mistake to remove rare SNPs $(\mathrm{MAF}<0.05)$ in association analysis. This was in contrast to the HapMap project in which the focus was on common SNPs. However, inclusion of rare SNPs resulted in higher location error for common disease Locus C (Table 2).

Occasionally or under high marker density, the kilobase map performed better than the LD map, presumably because every SNP has a unique physical location, whereas several SNPs could have the same LDU location in LD blocks. The tilted LD map improved the location accuracy for Locus $\mathrm{C}$, although not for Locus D (Table 2).

In practice, the phenomenon in this simulated data set may be too extreme. On the other hand, it is possible that several disease loci can be closely located. To distinguish such loci is a challenge to genetic epidemiologists. Under this circumstance, single SNP association plus a gene functional study may be useful.

Table I: Association mapping of disease Loci C and D on chromosome 6

\begin{tabular}{|c|c|c|c|c|c|c|}
\hline Region $^{\mathrm{a}}$ & No. SNPs & $S(k b)$ & $\Lambda_{\mathrm{A}}-\Lambda_{\mathrm{D}}$ & $\chi^{2}{ }_{3}$ & $P$ & $P_{\mathrm{c}}$ \\
\hline 26 & 128 & 24882 & 68 & 14 & $0.00247 \mid$ & 0.311346 \\
\hline 27 & 239 & 26121 & 793 & 40 & $<10^{-7}$ & 0.000001 \\
\hline 28 & 348 & 31299 & 5176 & 128 & $<10^{-12}$ & $<0.000001$ \\
\hline 29 & 176 & 32540 & 33058 & 322 & $<10^{-12}$ & $<0.000001$ \\
\hline 30 & 153 & 33962 & 295 & 25 & 0.000017 & 0.002129 \\
\hline 31 & 134 & 35638 & 103 & 15 & 0.001929 & 0.243096 \\
\hline 32 & 127 & 37776 & $14 \mid$ & 27 & 0.000007 & 0.000926 \\
\hline 33 & 147 & 39432 & 50 & 13 & 0.005554 & 0.699754 \\
\hline
\end{tabular}

aA segment of consecutive regions of 10 LDUs showing nominal significant association with RA $(p<0.05)$. See Methods for the meaning of other symbols. Loci $C$ and $D$ were in regions 29 and 32 at locations of 32485 and $37234 \mathrm{~kb}$, respectively.

${ }^{b} P_{c}$ is Bonferroni corrected $p$-value for multiple tests of 126 regions $(p \times 126)$. 
Table 2: Candidate regions of disease Loci C and D with rare SNPs included

\begin{tabular}{|c|c|c|c|c|c|c|c|}
\hline \multirow[b]{2}{*}{ Locus } & \multirow[b]{2}{*}{ Map } & \multirow[b]{2}{*}{$S(k b)$} & \multirow[b]{2}{*}{$\Lambda_{\mathrm{A}}-\Lambda_{\mathrm{D}}$} & \multirow[b]{2}{*}{$\chi_{3}^{2}$} & \multirow[b]{2}{*}{$p$} & \multicolumn{2}{|c|}{ Location error with rare SNPs } \\
\hline & & & & & & Included & Removed \\
\hline \multirow[t]{3}{*}{$\mathrm{C}$} & LD & 32557 & 30693 & 360 & $<10^{-12}$ & 72 & 55 \\
\hline & LD, tilt & 32518 & 30799 & 197 & $<10^{-12}$ & 34 & 21 \\
\hline & $\mathrm{kb}$ & 32506 & 28632 & 496 & $<10-12$ & 22 & 14 \\
\hline \multirow[t]{3}{*}{$\mathrm{D}$} & LD & 37358 & 154 & 22 & 0.000017 & 124 & 542 \\
\hline & LD, tilt & 37368 & 156 & 28 & 0.000003 & 130 & 546 \\
\hline & $\mathrm{kb}$ & 37368 & 148 & 17 & 0.000666 & 130 & 954 \\
\hline
\end{tabular}

\section{SNP density based on the order}

As density decreases, location error increases whether using single or multi-SNP approaches when the disease variant was not "typed" (Table 3 ). There was an improvement in accuracy when the disease variant was included. In most cases, using the LD map resulted in greater accuracy than using the kilobase map, especially when the marker density was low. We also selected SNPs on the scale of one to the hundredth or even the thousandth. As long as there was one SNP highly associated with the disease (e.g., $\chi^{2}{ }_{1}=27$ ), the association was detectable, but much compromised by precision as a result of low SNP density. These data are unusual in that the association of Locus $\mathrm{C}$ is extremely significant and probably would not be observed in the real data.

Although mapping accuracy decreases with marker density, even with $1 / 30$ the number of SNPs, corresponding to a $10 \mathrm{~K}$ GWA scan, we could still detect Locus C (Table $3)$. Single SNP tests depend heavily on whether the disease variant is typed. It has less predictive value for accuracy because the SNP with maximal $\chi^{2}$ is not necessarily the closest SNP to the disease variant. In contrast, meth- ods that combine information from multiple markers predict the location of the disease variant better than single SNP tests because the location is less influenced by any single SNP effects. A multi-marker approach may therefore be more robust to genotyping errors.

We expect that the mapping accuracy will be improved further in maps with higher marker density than that assessed in this paper, such as the commercially available $500 \mathrm{~K}$ or more genotyping platforms for GWA studies.

\section{SNP density based on tagging or equidistance}

For the $15,805.710 \mathrm{~kb}$ candidate region spanning both Loci C and D, we compared location accuracy using SNPs selected with Tagger or by equidistance of LDU or kilobases (Table 4). SNPs based on equal LDU provided higher location accuracy than those based on equal kilobase distance. Equidistance generally provided higher accuracy than tagging SNP selection. Again, reducing SNP density decreases the prediction accuracy of disease Loci $\mathrm{C}$ and $\mathrm{D}$, but this was minimally affected by selection based on equal LD distance (Table 4).

Table 3: Density and accuracy for Locus C - SNP selection by order

\begin{tabular}{|c|c|c|c|c|c|c|c|c|}
\hline \multirow[b]{3}{*}{ SNP density (kb/SNP) } & \multirow[b]{3}{*}{ No. SNPs } & \multirow[b]{3}{*}{ No. regions } & \multicolumn{2}{|r|}{ msSNP } & \multicolumn{4}{|c|}{ Location error by the composite likelihood approach } \\
\hline & & & \multirow[b]{2}{*}{$\chi_{1}^{2}$} & \multirow[b]{2}{*}{ Location error } & \multicolumn{2}{|c|}{ Causal SNP out } & \multicolumn{2}{|c|}{ Causal SNP in } \\
\hline & & & & & LD & $\mathrm{kb}$ & LD & $\mathrm{kb}$ \\
\hline Full (II) & $|570|$ & 126 & 2324 & 153 & 57 & 13 & 55 & 14 \\
\hline $\mathrm{I} / 2(22)$ & 7850 & 125 & 1762 & -2 & 5 & -19 & 5 & -20 \\
\hline I/3 (33) & 5233 & 118 & 2324 & 153 & 153 & 40 & 6 & 40 \\
\hline I/4 (44) & 3925 & 106 & 1762 & -2 & -65 & -56 & -57 & -53 \\
\hline I/5 (54) & 3140 & 94 & 2274 & 42 & -24 & -35 & -15 & -36 \\
\hline $1 / 6(65)$ & 2616 & 82 & 1285 & 20 & 20 & 20 & 10 & 15 \\
\hline I/8 (87) & 1962 & 64 & $160 \mid$ & 65 & -58 & -64 & -47 & -58 \\
\hline $1 / 10(109)$ & 1570 & 52 & 726 & -106 & -55 & -59 & -24 & -46 \\
\hline $1 / 15(163)$ & 1046 & 34 & 486 & -887 & 294 & 26 & 0 & 3 \\
\hline I/20 (2I7) & 785 & 26 & 726 & -106 & -97 & -160 & -26 & -43 \\
\hline I/25 (272) & 628 & 20 & 348 & -9 & 69 & -120 & 60 & -79 \\
\hline $1 / 30(326)$ & 523 & 17 & 229 & 188 & 362 & -25 & 0 & -25 \\
\hline
\end{tabular}

Disease variant $C\left(\chi_{1}^{2}=1916\right)$ was not present except in the full data set or specified. 
Table 4: Density and accuracy - SNP selection by tagging or equidistance ${ }^{a}$

\begin{tabular}{|c|c|c|c|c|c|c|c|}
\hline \multirow[b]{2}{*}{$R^{2}(\mathrm{LDU}, \mathrm{kb})$} & \multirow[b]{2}{*}{ No. SNPs (kb/SNP) } & \multicolumn{3}{|c|}{ Locus C } & \multicolumn{3}{|c|}{ Locus D } \\
\hline & & Tagger & E_LD & E_kb & Tagger & E_LD & E_kb \\
\hline Full & $1658(10)$ & 20 & 20 & 20 & 130 & 130 & 130 \\
\hline $0.8(0.013,7)$ & $1080^{\mathrm{b}}(15)$ & 35 & 20 & 30 & 130 & 124 & 130 \\
\hline $0.6(0.025,10)$ & $874(18)$ & 56 & -16 & 27 & 545 & 124 & 551 \\
\hline $0.4(0.047,15)$ & $657(24)$ & 106 & 42 & 63 & 124 & 123 & 117 \\
\hline $0.2(0.099,27)$ & $421(38)$ & 125 & 14 & -23 & 537 & 112 & 231 \\
\hline
\end{tabular}

aLocation error for SNPs selected by Tagger or equal LDU (E_LD) or kb (E_kb) distance in a candidate region of I $5805.710 \mathrm{~kb}$ with rare SNPs included. Regions were fixed at 10 LDUs for Loci C (30997-33398 kb) and D (36784-37792 kb). Disease variant C was not present except in the full data set. Tilted LD map.

bl 079 for E_LD.

\section{Sample size and region length}

We analyzed different sample sizes based on the combination of 500, 1000, 1500, and 2000 cases or controls. Despite variations in location errors for Locus C, there was no clear trend to draw any meaningful conclusion. For Locus $\mathrm{D}$, however, a high degree of accuracy appeared to be maintained when the data sets had over 1000 cases and 1500 controls. Therefore, large samples are needed for detecting rare disease loci.

With Locus $C$ being centred, we studied region lengths from 0.2 up to 30 LDUs, with the latter starting in region 27 and ending in region 30 . The location error was relatively stable but extremely small or large LDU lengths resulted in increased error. The region lengths in LDUs (location errors in kilobases) were 0.2 (107), 1 (5), 2 (82), 4 (5), 6 (5), 8 (5), 10 (5), $12(-10), 14(-10), 16(-13), 18$ $(-14), 20(-14)$, and $30(-68)$. We therefore recommend $10-\mathrm{LDU}$ for the maximal length while maintaining minimal error. Increasing the number of SNPs also linearly increases the computing load [3].

Fixing region length had no appreciable impact on location accuracy at high density, but the errors were greater than let-the-program-decide regions at low density (data not shown).

\section{Conclusion}

We successfully detected disease Loci C and D in the simulated dense chromosome 6 data using the Malecot model and composite likelihood approach. Decreasing SNP density compromises accuracy of association mapping. This multi-marker approach has many advantages. Firstly, it markedly decreases the number of tests in GWA studies, avoiding heavy penalty for multiple testing. Secondly, it predicts the disease loci more accurately than single SNP association tests. We also demonstrated that SNP selection by equal LD distance outperforms that by tagging or equal kilobase distance in the accuracy of association mapping. Finally, we conclude that excluding rare
SNPs significantly decreases the power and accuracy in mapping rare disease loci.

\section{Competing interests}

The author(s) declare that they have no competing interests.

\section{Acknowledgements}

WZ was supported by the Institute of Cancer Research, Sutton, Surrey, UK. WL, CH, and T-YK were supported by Ph.D. studentships funded by the University of Southampton, Shanghai Jiaotong University, and the Taiwan Ministry of Education, respectively.

This article has been published as part of BMC Proceedings Volume I Supplement I, 2007: Genetic Analysis Workshop I5: Gene Expression Analysis and Approaches to Detecting Multiple Functional Loci. The full contents of the supplement are available online at http://www.biomedcentral.com/ $|753-656| / \mid$ ? issue=SI.

\section{References}

I. Morton NE, Maniatis N, Zhang W, Ennis S, Collins A: Genome scanning by composite likelihood. Am J Hum Genet 2007, 80:19-28.

2. Gomes I, Collins A, Lonjou C, Thomas NS, Wilkinson J, Watson M, Morton N: Hardy-Weinberg quality control. Ann Hum Genet 1999, 3:535-538.

3. Lau W, Kuo TY, Tapper W, Cox S, Collins A: Exploiting large scale computing to construct high resolution linkage disequilibrium maps of the human genome. Bioinformatics 2007, 23:517-519.

4. Carlson CS, Eberle MA, Rieder MJ, Yi Q, Kruglyak L, Nickerson DA: Selecting a maximally informative set of single-nucleotide polymorphisms for association analyses using linkage disequilibrium. Am J Hum Genet 2004, 74: 106-I 20. 